\title{
Vascularização arterial da região do nó sinoatrial em corações suínos: origem, distribuição e quantificação'
}

\author{
Ana P. Vidotti ${ }^{*}$, Fernanda R. Agreste ${ }^{3}$, Pedro P. Bombonato ${ }^{4}$, Isaura M. M. \\ Prado $^{5}$ e Rosângela Monteiro 6
}

\begin{abstract}
Vidotti A.P., Agreste F.R., Bombonato P.P. \& Prado I.M.M. 2008. [Arterial vascularization of the sinoatrial node in swine hearts: origin, distribution and quantification.] Vascularização arterial da região do nó sinoatrial em corações suínos: origem, distribuição e quantificação. Pesquisa Veterinária Brasileira 28(2):113-118. Departamento de Cirurgia, Setor de Anatomia dos Animais Domésticos e Silvestres, Faculdade de Medicina Veterinária e Zootecnia, Universidade de São Paulo, Av. Prof. Dr. Orlando Marques de Paiva 87, Cidade Universitária, São Paulo, SP 05508-000, Brazil. E-mail: avidotti@usp.br

The sinoatrial node, for being topographically installed as the initial component of the conduction system, is responsible for the production of the nervous impulses, which determines the cardiac contraction. There have been made studies related to the node's morphology in order to know the origin, distribution and quantification of the vases in this tissue, however, in spite of the results and quantitative data of the nodal irrigation - arterial vascular conduct and arterial vascular density, at the nodal level - the literature is scarce. With this objective 27 SRD swine hearts, injected with colored resin for macroscopic analysis of the origin and distribution of ANSA (sinoatrial node artery), 3 others injected with watery solution of colloidal coal (dyed nanquim) were used, to mark the route of the vases on the node level for stereological analysis. The atrial arteries originated as well from the right coronary artery as from the left one, with predominance of the first $(66.66 \%$ and $33.33 \%$, respectively). When originated from the right coronary artery, there existed the following branches: AADAM (right cranial medial atrial artery) in 14 cases, AADAI (right cranial intermedial atrial artery) in 2 cases, and AADAL (right cranial lateral atrial artery) in 2 cases. In 9 cases (33.33\%) the following branches originated from the left coronary artery: 4 through branch AASPL (left caudal lateral atrial artery), 2 through branch AASAI (left cranial intermedial atrial artery) and 3 through branch AASAM (left cranial medial atrial artery). Interarterial anastomoses with vessels responsible for irrigation of the sinoatrial node area were observed in most of the cases (25 hearts). The Volume or Reference Volume $\left(\mathrm{V}\left({ }_{\text {ref }}\right)\right)$ of the hearts were $35.32 \times 10^{4} \mu \mathrm{m}^{3}$. For the stereological variables analyzed, the estimate density of the vessel length (LV) was 766; the vessel length (L) - mm - was $27.06 \times 10^{5} \mu \mathrm{m}$; the area's surface density (Sv) was 182 and the area's surface (S) - $\mathrm{mm}^{2}$ - was $64.3 \times 10^{6} \mu \mathrm{m}^{2}$. The estimate of the vascular numeric density $\left(\mathrm{Nv}\left({ }_{\text {vasc }}\right)\right)$ - total of vessels per unit of volume $\left(\mathrm{cm}^{3}\right)$ was $2.1910^{-5}$, and the total number of vessels in the organ $\left(\mathrm{N}_{\text {vasc }}\right)$ ), estimated by the physical dissector method in combination with the estimate of the number of Euler (Xv), was $773.6832 \times 10^{-2}$. The raised vascular density and total number of vases in the swine's sinoatrial node area suggests the
\end{abstract}

\footnotetext{
${ }^{1}$ Recebido em 12 de junho de 2007.

Aceito para publicação em 15 de fevereiro de 2008.

${ }^{2}$ Bolsista de doutorado da FAPESP. Endereço: Rua Prof. Guido Inácio Bersch 60, apt. 4, Jardim Universitário, Maringá, PR 87020-250, Brasil. *Autor para correspondência: avidotti@usp.br

${ }^{3}$ Pós-Graduanda do Departamento de Cirurgia, Faculdade de Medicina Veterinária e Zootecnia (FMVZ), Universidade de São Paulo (USP), São Paulo, SP, Brasil.
}

\footnotetext{
${ }^{4}$ Departamento de Cirurgia , FMVZ, USP.

${ }^{5}$ Departamento de Ciências Morfofisiológicas, Universidade Estadual de Maringá. Av. Colombo 5790, Campus Universitário, Maringá, PR 877045-000.

${ }^{6}$ Instituto do Coração, Hospital das Clínicas, Universidade de São Paulo (INCOR/HC-USP), Av. Dr. Enéas de Carvalho Aguiar 44, ํㅡ andar, Cerqueira, São Paulo, SP 05403-001.
} 
existence of a vascular perinodal network complex and dense, ratifying the importance of this mark-pass for its blood supply.

INDEX TERMS: Heart, vascularization, sinoatrial node, morphometry, swine.

RESUMO.- O nó sinoatrial, por se encontrar topograficamente instalado como componente inicial do sistema de condução, é responsável pela geração dos impulsos nervosos determinantes da contração cardíaca. Estudos relacionados à morfologia do nó, visando conhecer a origem, trajeto e distribuição dos vasos neste tecido são conhecidos, contudo, no que diz respeito a estes aspectos e aos dados quantitativos da irrigação nodal, no que se refere ao comportamento vascular arterial e a densidade vascular arterial desta região, a literatura é escassa. Com este objetivo foram utilizados 30 corações de suínos SRD, sendo 27 injetados com resina vinílica corada, para análise da origem e trajeto da ANSA (artéria do nó sinoatrial) e 3 corações injetados com solução aquosa de carvão coloidal (tinta nanquim) para proceder à análise estereológica. As artérias atriais originaram-se tanto da artéria coronária direita quanto da esquerda, com predominância da primeira $(66,66 \%$ e $33,33 \%$, respectivamente). Quando originada da coronária direita, a irrigação ocorreu pelo ramo AADAM (artéria atrial direita cranial medial) em 14 casos e pelos ramos AADAI (artéria atrial direita cranial medial) em 2 casos e AADAL (artéria atrial direita cranial lateral) em 2 casos. Em 9 casos (33,33\%) originou-se pela artéria coronária esquerda: quatro pelo ramo AASPL (artéria atrial esquerda caudal lateral), dois pelo ramo AASAI (artéria atrial esquerda cranial intermédia) e três pelo ramo AASAM (artéria atrial esquerda cranial medial). Anastomoses interarteriais, com participação dos vasos responsáveis pela irrigação do território do nó sinoatrial foram observadas na maioria dos casos (25 corações). O Volume do órgão ou Volume Referência $\left.\left(V_{\text {ref }}\right)\right)$ foi de $35,32 \times 10^{4} \mu \mathrm{m}^{3}$. Para as variáveis estereológicas analisadas, a estimação da densidade de comprimento do vaso (Lv) foi de 766; o comprimento do vaso $(L)$ - $\mathrm{mm}$ - foi de $27,06 \times 10^{5} \mu \mathrm{m}$; a densidade de superfície de área (Sv) foi de 182 e a superfície de área (S) - $\mathrm{mm}^{2}$ - foi de $64,3 \times 10^{6} \mu \mathrm{m}^{2}$. A estimação da densidade numérica vascular $\left(\mathrm{N}_{\mathrm{v}(\text { vasc })}\right)$, quantidade de vasos por unidade de volume $\left(\mathrm{cm}^{3}\right)$, foi de $2,1910^{-5}$ e o número total de vasos no órgão $\left(\mathrm{N}_{\text {(vasc })}\right)$, estimado pelo método dissector físico em combinação com a estimativa do número de Euler $\left(X_{v}\right)$, foi de $773,6832 \times 10^{-2}$. A elevada densidade vascular e do número total de vasos na região do nó sinoatrial de suínos sugere a existência de uma complexa e densa rede vascular perinodal, ratificando a importância deste marca-passo pelo seu suprimento sangüíneo.

TERMOS DE INDEXAÇÃO: Coração, vascularização, nó sinoatrial, morfometria, suíno.

\section{INTRODUÇÃO}

O nó sinoatrial (NSA), descrito por Keith e Flack em 1907, localizado entre o átrio direito e a desembocadura da veia cava cranial, é considerado o "marca-passo" do coração, porquanto coordena o seu sistema excito-condutor. O NSA consiste de uma região com fibras musculares dispersas e pálidas, pobres em miofibrilas, entremeadas por fibras colágenas, elásticas e reticulares e tecido nervoso, de difícil evidenciação macroscópica (Glomset \& Glomset 1940, Halpern 1955). Está intimamente associado com uma artéria bem definida - artéria do nó sinoatrial - ANSA, seu principal suprimento arterial, em geral, com origem unilateral (James 1961) e ponto de referência para a localização deste nó em mamíferos (Keith \& Flack apud Melo 2000, James 1961, Mandarim-de-Lacerda 1990).

A importância funcional dessa estrutura tem levado vários pesquisadores a se interessarem pelos aspectos morfológicos e funcionais desse marca-passo e seu suprimento sanguíneo em várias espécies animais como, por exemplo, em cães (Pina et al. 1975, Randi 1988, Ovèina 2002), em bovinos (Borelli 1968, Caetano 1982, Severino 1986, Caetano et al.1995b, Ovèina 2002), em babuínos (Mandarim-de-Lacerda \& Hureau 1986), em suínos (Weaver et al. 1986, Severino et al. 2000), em ratos (Halpern 1955, Melo-da-Silva \& Mandarim-de-Lacerda 1994, Melo 2000, Melo et al. 2002) e mesmo no homem (Kennel \& Titus 1972 Jatene et al. 1999, Berdajs et al. 2003). Contudo, no que diz respeito ao trajeto da ANSA, sua terminação junto ao NSA, e aos aspectos quantitativos da vascularização do nó a literatura é escassa.

Os suínos, além de sua destacada importância na área de produção de proteína de origem animal, têm-se mostrado como animais de eleição para estudos comparativos de vários órgãos e sistemas, pela similitude com a espécie humana e, portanto, aspectos de sua morfologia precisam ser melhores estudados para propiciar elementos e subsídios para a comparação e inferência de resultados.

Dentre os estudos morfológicos que podem nortear a análise comparativa os estudos estereológicos são cada vez mais freqüentes na literatura, particularmente nos campos do desenvolvimento, evolução, patologia e neurociências. $O$ desafio da estereologia é interpretar 0 arranjo estrutural tridimensional interno, com base na análise quantitativa de porções da estrutura que mostram apenas uma informação bidimensional, tendo como princípio considerações geométricas e probabilidade estatística (Weibel 1979, Jensen 1998, Mandarim-de-Lacerda 2003). A estereologia destaca-se entre os tipos de análises existentes na literatura, por apresentar resultados numéricos e não subjetivos, por facilitar a comparação entre diferentes grupos em algumas situações (idade, espécie, etc), por apresentar estratégia de amostragem estrita, por ser considerado um método aceitável, além do baixo custo do equipamento (Bolenger 1981, 1982, Gundersen et al. 1999, Bjarkam et al. 2001, Kubinova et 
al. 2001, Mandarim-de-Lacerda \& Pereira 2001, Charleston et al. 2003, Davey et al. 2003).

Baseados nestes pressupostos, este trabalho objetivou estudar a vascularização arterial da região do NSA, em corações suínos SRD, por meio de técnicas macroscópicas e estereológicas. Procuramos enfocar, em especial, a terminação da ANSA, sua origem e arranjo, bem como conhecer o comportamento vascular arterial e a densidade vascular arterial desta região, na tentativa de verificar a existência de uma rede vascular perinodal indicada na literatura para algumas espécies (Vidotti 2006).

\section{MATERIAL E MÉTODOS}

Foram utilizados 30 corações de suínos, machos, SRD, provenientes do Frigorífico FERMAR, Município de Floraí, Paraná. Após isolamento do órgão e canulação dos vasos, as artérias coronárias de 27 corações, foram injetadas com resina vinílica corada para análise macroscópica da origem e trajeto da ANSA; e de 3 corações com solução aquosa de carvão coloidal (tinta nanquim) para proceder à análise estereológica da vascularização do NSA.

Após injeção com resina vinílica, os corações foram fixados em solução aquosa de formol a $10 \%$ por no mínimo 48 horas, para posterior dissecação da circulação atrial, seguindo o trajeto das artérias, procurando-se identificar a origem e trajeto da artéria do nó sinoatrial. A nomenclatura adotada para identificação e descrição das artérias atriais baseou-se no proposto por DiDio \& Wakefield (1972) para o homem, com a adaptação da terminologia em função da posição quadrúpedal dos animais em questão, qual seja: AADAM (artéria atrial direita cranial medial); AADAI (artéria atrial direita cranial intermédia); AADAL (artéria atrial direita cranial lateral); AADPL (artéria atrial direita caudal lateral); AADPI (artéria atrial direita caudal intermédia); AADPM (artéria atrial direita caudal medial); AASPM (artéria atrial esquerda cranial medial); AASPI (artéria atrial esquerda cranial intermédia); AASPL (artéria atrial esquerda caudal lateral); AASAL (artéria atrial esquerda cranial lateral); AASAI (artéria atrial esquerda cranial intermédia); AASAM (artéria atrial esquerda cranial medial).

Os três corações injetados com solução aquosa de carvão coloidal tiveram a região do nó sinoatrial identificada e retirada para preparação, dentro dos procedimentos convencionais da rotina histológica, para inclusão em parafina. Os blocos contendo a região nodal foram trimados para evidenciar a região de interesse e seccionados seriadamente, com cortes de $5 \mu \mathrm{m}$ de espessura, no sentindo ápice para base do coração, e corados pelas técnicas de Hematoxilina de Van Gieson, Tricrômio de Gomori e Weigert - Van Gieson para observação sob microscópio de luz.

A análise quantitativa tridimensional foi realizada utilizando o método estereológico do disector físico, descrito por Sterio (1984) e adaptado por Pakkenberg \& Gundersen $(1988,1989)$, associado ao princípio de ConnEuler. O método do disector foi utilizado para contar as estruturas independentemente do tamanho, forma, distribuição ou orientação, em um volume conhecido, utilizando pares de secções provenientes de cortes seriados e permitiu a composição das estruturas em três dimensões, utilizando um sistema teste com uma área (a) conhecida, número de pontos (p), e um plano paralelo com uma altura (h) (Agreste 2005). O princípio de ConnEuler ou de conectividade, que é uma extensão do método de disector, foi utilizado para a estimativa do número de vasos, onde cada vaso tem um número de Euler ou conectividade que foi obtido com secções seriadas da amostra (Kroustrup \& Gundersen 2001). A partir desses dados, foram obtidos os valores estereológicos de densidade vascular, comprimento vascular, densidade de superfície, superfície de área vascular e número total de vasos.

À semelhança dos procedimentos adotados para a área nodal examinou-se também a área atrial diametralmente oposta à região do nó de um coração, no sentido de propiciar a análise comparativa dos valores e para estabelecimento de valores padrões.

Por fim, confrontaram-se os valores relativos à densidade vascular e o número total de vasos entre as regiões do nó e a área atrial oposta, com o auxílio do Teste do Qui-quadrado com nível de significância de 5\%.

\section{RESULTADOS}

A injeção de resina vinílica, seguida de dissecação, permitiu a identificação das artérias atriais, bem como a origem da artéria nodal e, em alguns casos, de seu trajeto até a região do nó sinoatrial. As artérias atriais originaram-se tanto da artéria coronária direita quanto da esquerda: em 18 casos $(66,66 \%)$ originou-se da artéria coronária direita, sendo que em 14 destes, sua emergência se deu pelo ramo atrial direito cranial medial (AADAM); em dois casos, do ramo atrial direito cranial intermédio (AADAI), e em outros dois do ramo atrial direito cranial lateral (AADAL). Em 9 casos (33,33\%), a ANSA originou-se da artéria coronária esquerda pelo ramo atrial esquerdo caudal lateral (AASPL) em 4 casos, pelo ramo atrial esquerdo cranial intermédio (AASAI) em dois casos, e pelo ramo atrial esquerdo cranial medial (AASAM) em três corações. Anastomoses interarteriais, com participação dos vasos responsáveis pela irrigação do território do nó sinoatrial foram observadas na maioria dos casos (25 corações). Contudo não foi possível distinguir, com precisão, as artérias que as constituíam (Fig.1 e 2).

$\mathrm{O}$ Volume do órgão ou Volume Referência $\left(\mathrm{V}_{(\mathrm{ref})}\right) \mathrm{cm}^{3}$ para o segmento contendo o nó sinoatrial foi estimado

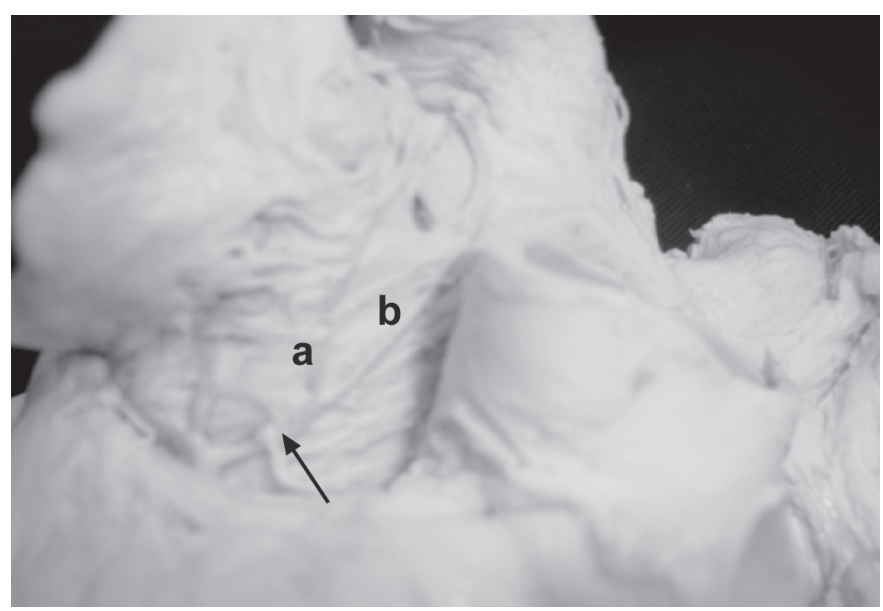

Fig.1. Vista da base de coração suíno. Artéria atrial direita cranial medial AADAM (seta) emitindo ramos $(a, b)$ para a região do nó sinoatrial (originando a ANSA). 


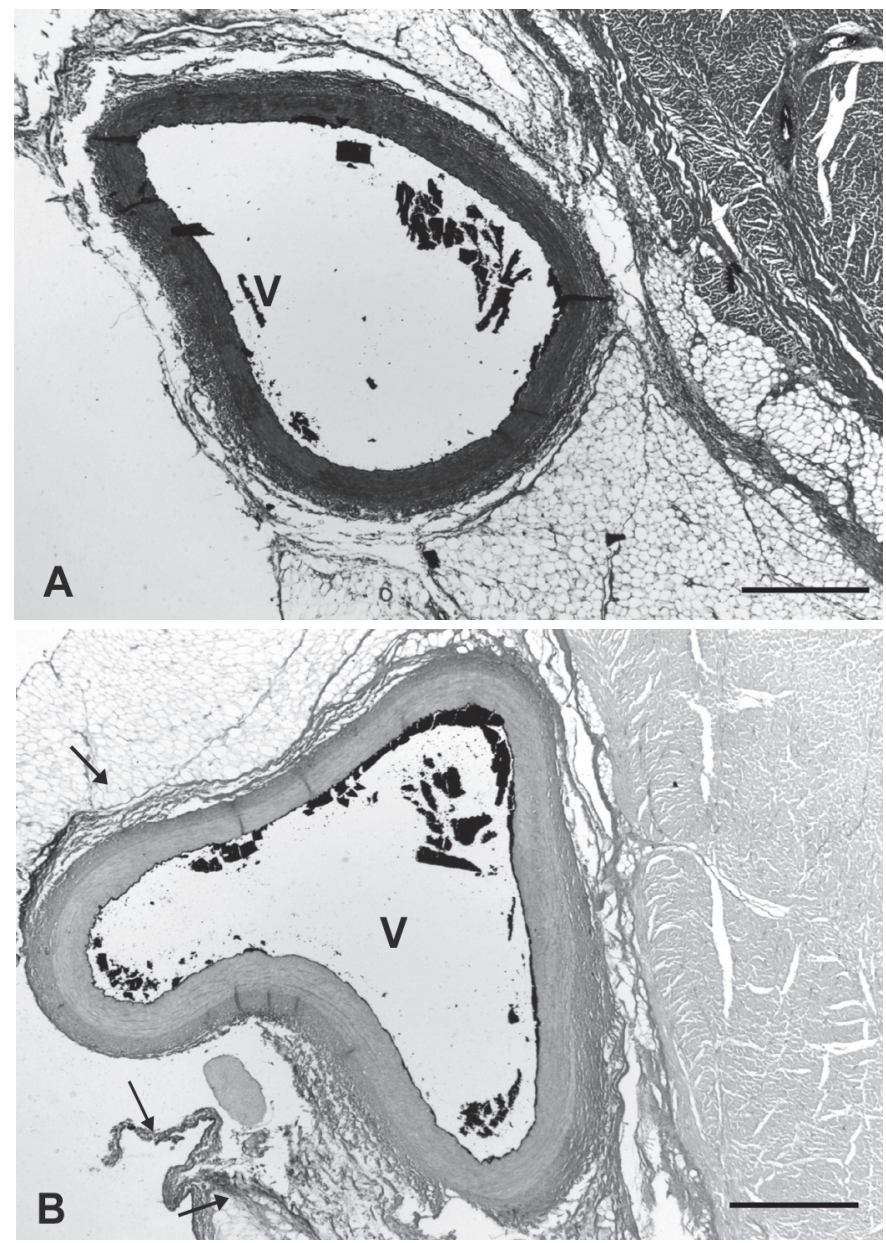

Fig.2. Região do NSA de coração de suíno, evidenciando vaso confluente para a formação da ANSA. (A) Fibras colágenas (em azul), fibras musculares (em vermelho). (B) Fibras colágenas (em vermelho), fibras musculares (em laranja) e fibras elásticas (setas). (A) Tricrômio de Gomori, (B) WeigertVan Gieson. Barra: 500um.

em $35,32 \times 10^{4} \mu m^{3}$ pelo método de Cavalieri através de planos macroscópicos paralelos e seriados. Para as variáveis estereológicas analisadas, a densidade de comprimento do vaso (Lv) foi estimada em 766 ; o comprimento do vaso (L) - mm - foi de $27,06 \times 10^{5} \mu \mathrm{m}$; a densidade de superfície de área (Sv) foi de 182 e a superfície de área (S) - $\mathrm{mm}^{2}$ - foi de $64,3 \times 10^{6} \mu \mathrm{m}^{2}$.

A estimação da densidade numérica vascular $\left(\mathrm{N}_{v \text { (vasc }}\right)$, quantidade de vasos por unidade de volume $\left(\mathrm{cm}^{3}\right)$, foi de $2,19 \times 10^{-5}$ e o número total de vasos no órgão $\left(\mathrm{N}_{(\text {vasc }}\right)$, estimado pelo método disector físico em combinação com a estimativa do número de Euler $\left(X_{v}\right)$, foi de $773,6832 \times 10^{-2}$.

A análise estatística apoiada no Teste do Qui-quadrado evidenciou existir diferenças entre os valores da densidade vascular e do número total de vasos entre a região do nó e a área atrial diametralmente oposta.

\section{DISCUSSÃO}

Muitas técnicas anatômicas são propostas para o estudo do sistema vascular e a seleção da técnica mais adequada envolve vários fatores (Jatene et al. 1999). Na busca daquela que oferecesse maior definição e alcance para o estudo macroscópico da origem e distribuição da ANSA ao nível do NSA, optamos pela técnica de injeção de resina vinílica (vinilite), seguida de dissecação, a qual, ainda que não atinja os vasos de calibres diminutos e, portanto, a caracterização dos ramos anastomóticos, permite a visibilização do trajeto da ANSA na região do NSA, conservando sua relação com as demais estruturas anatômicas atriais, à semelhança do observado por Jatene et al. (1999) e que para nosso estudo era de fundamental importância para a condução do estudo estereológico e a demarcação da região do nó sinoatrial.

A ANSA de suínos originou-se, na maioria dos casos, da artéria coronária direita, confirmando o observado para esta espécie (Weaver et al. 1986, Severino et al. 2000) e para o homem (James 1961, 1977, Nerantzis \& Avgoustakis 1980, Kyriakidis et al. 1983, Eliska \& Eliskova 1990, Caetano et al. 1995a, DiDio et al. 1995, Jatene et al. 1999, Berdajs et al. 2003), ainda que com freqüências diferentes daquela por nós identificadas, sugerindo que a artéria coronária direita, com diferentes graus de participação, responde por significativa parcela na nutrição do SNA, independentemente da espécie animal .

Observamos que o principal ramo que originava a ANSA provinha da artéria atrial direita cranial medial AADAM, concordando com o apontado, para a espécie humana, por Caetano et al. (1995a) e Di Dio et al. (1995). Em alguns casos, observamos a origem da AADAL e AADAl (2 casos cada) não indicada por aqueles autores. A origem da ANSA a partir de ramos correspondentes a AADAM foi, também, indicada por outros autores, ainda que tenham adotado nomenclatura diversa: para suínos (Weaver et al. 1986, Severino et al. 2000) e para o homem (Krupa 1993, 1995, Sow et al. 1996, Jatene et al. 1999, Berdajs et al. 2003).

Em alguns casos, a origem da ANSA se dava a partir da artéria coronária esquerda, confirmando o observado também para suínos (Severino et al. 2000, Weaver et al. 1986), para cães (Pina et al. 1975, Randi 1988, Ovèina, 2002), bovinos (Borelli 1968, Caetano 1982, Caetano et al. 1995b, Severino 1986, Ovèina 2002), babuínos (Mandarim-de-Lacerda \& Hureau 1986) e para o homem (James 1961, 1977, Nerantzis \& Avgoustakis 1980, Kyriakidis et al. 1983, Eliska \& Eliskova 1990, Caetano et al. 1995a, DiDio et al. 1995, Jatene et al. 1999, Berdajs et al. 2003).

Em nossos resultados, os ramos provenientes da artéria coronária esquerda, que originaram a ANSA foram, principalmente, a artéria atrial esquerda caudal lateral $(A A S P L)$ e a artéria atrial esquerda cranial medial (AASAM), concordando com o indicado para esta mesma espécie, mas em animais de padrão racial diferente, estudados por Severino et al. (2000) e Weaver et al. (1986). Este padrão foi, também, verificado em corações de indivíduos humanos, por Caetano et al. (1995a), DiDio et al. 
(1995), Futami et al. (2003) e Kawashima \& Sasaki (2003), os quais adotaram nomenclatura semelhante a nossa; e por Kennel \& Titus (1972), Kyriakidis et al. (1983), Busquet et al. (1984), Eliska \& Eliskova (1990), Krupa (1993,1995), Sow et al. (1996), Jatene et al. (1999) e Berdajs et al. (2003) que adotaram nomenclatura diferenciada.

O curso das artérias atriais até a formação da ANSA apresentou-se variável na origem e no seu trajeto, não sendo possível identificar um "padrão vascular" para a artéria do nó sinoatrial. Nossos resultados evidenciaram anastomoses interatriais na região do nó, concordando com o observado por outros autores, para suínos (Weaver et al. 1986, Severino et al. 2000); bovinos (Borelli 1968, Caetano 1982, Severino 1986, Caetano et al. 1995b, Ovèina 2002) e para o homem (James (1961), Kennel \& Titus (1972), James (1977), Nerantzis et al. (1980), Eliska \& Eliskova (1990), Krupa (1993, 1995), Sow et al. (1996), Lopes et al. (1998) e Kawashima \& Sasaki (2003). A julgar pela importância funcional da estrutura em questão, podemos esperar um arranjo específico, com grande número de anastomoses, no tecido nodal, de modo a preservar a fisiologia cardíaca, como já foi apontado por diversos autores para o homem (Truex et al. 1967, Mandarim-de-Lacerda et al. 1985, Caetano et al. 1995a, DiDio et al. 1995, Ovèina \& Æemerliæ 1997, Lopes et al. 1998).

De fato, foi possível evidenciar grande densidade vascular e de número total de vasos na região do nó sinoatrial de suínos, o que pode sugerir a existência de uma rede vascular perinodal como insinuado por alguns autores. O método de Cavalieri e o coeficiente de erro utilizado para obtenção do volume do órgão neste trabaIho, apresentaram eficiência comprovada em estudos de outros órgãos e tecidos por Gundersen \& Jensen (1987), Mayhew (1992), Duerstock et al. (2003), Wulfsohn et al. (2004) e Agreste (2005), o que salienta que a metodologia utilizada na caracterização dos valores estereológicos possui confiabilidade e que, portanto, transmite esse mesmo grau de confiabilidade a sugestão da propositura da rede perinodal no nó sinoatrial de suínos.

Os dados obtidos sugerem uma intensa vascularização da região do nó sinoatrial de suínos, o que pode contribuir para o entendimento de questões morfológicas gerais de comportamento vascular e estrutural dessa estrutura. A fisiologia do NSA e a literatura específica (Truex et al. 1967, Mandarim-de-Lacerda et al.1985, Caetano et al. 1995a, DiDio et al. 1995, Ovèina \& Æemerliæ 1997, Lopes et al. 1998) sugerem a necessidade de um maior aporte sangüíneo nesta região do que nas demais regiões atriais. Assim, estudos estereológicos comparativos entre esta e as demais regiões atriais, particularmente com aquela oposta ao NSA, podem contribuir para elucidar a distribuição da vascularização da região do NSA nas várias espécies domésticas. Os resultados desses estudos contribuem para o entendimento do funcionamento deste importante marca-passo cardíaco e podem ser correlacionados com diversos fatores, incluindo o sexo, a idade.
Essas análises geram informações importantes na elucidação das inocorrências de alterações significativas do ritmo cardíaco em pacientes com bloqueios da ANSA, sugerindo que a irrigação do nó, nestes casos, poderia ser mantida, pelo menos em parte, por aquela grande densidade vascular própria da região como sugerido por Vidotti (2006) para o homem.

\section{CONCLUSÕES}

A técnica de injeção de vinilite evidenciou a origem e a distribuição da artéria do nó sinoatrial de suínos, as quais apresentaram grande semelhança com aquelas observadas em corações de humanos e de outras espécies animais, com predominância da artéria coronária direita sobre a esquerda. A avaliação estereológica, pelas técnicas empregadas, mostrou uma elevada densidade vascular e do número total de vasos na região do nó sinoatrial de suínos indicando a existência de uma complexa e densa rede vascular perinodal, ratificando a importância deste marca-passo pelo seu suprimento sangüíneo.

\section{REFERÊNCIAS}

Agreste F.R. 2005. Estudo quantitativo da vascularização do timo em cães. Dissertação de Mestrado, Faculdade de Medicina Veterinária e Zootecnia, Universidade de São Paulo, São Paulo. 118p.

Bjarkam C.R., Pedersen M. \& Sorensen S. 2001. New strategies for embedding orientation and sectioning os small brain specimens enable direct correlation to MR-Images, brain atlases, or use of unbiased stereology. J. Neurosci. Methods 108:153-159.

Berdajs D., Patonay L. \& Turina M.I. 2003. The clinical anatomy of the sinus node artery. Ann. Thorac. Surg. 76(3):732-736.

Bolenger R.P. 1981. Stereology: applications to pharmacology. Annu. Rev. Pharmacol. Toxicol. 21:549-573.

Bolenger R.P. 1982. Biological stereology: history, present state, future directions. Microscop. Res. Tech. 21:255-261.

Borelli V. 1968. Contribuição ao estudo da irrigação arterial do nó sinuatrial em bovinos azebuados. Tese de Doutorado, Faculdade de Medicina Veterinária e Zootecnia, Universidade de São Paulo, São Paulo. $55 p$.

Caetano A.G. 1982. Contribuição ao estudo da irrigação arterial do Nodus sinuatrialis em bovinos da raça Gir. Dissertação de Mestrado, Escola Paulista de Medicina, Universidade de São Paulo, São Paulo. 63p.

Caetano A.G., Lopes A.C., DiDio L.J.A. \& Prates J.C. 1995a. Critical analysis of the clinical and surgical importance of the variations in the origin of the sinoatrial node artery of the human heart. Revta Assoc. Med. Bras. 41(2):94-102.

Caetano A.G., Borelli V., Prates J.C. \& DiDio L.J.A. 1995b. The arterial supply of the Nodus sinuatrialis in bovine Gir. Braz. J. Morphol. Sci. 12(2):81-88.

Charleston L.B., Thyer A.C., Klein N.A., Soules M.R. \& Charleston J.S. 2003. An improved method for the production of slides from oversized sample of glycol methacrylate-embedded tissue: application for optical dissector based stereology. J. Histotechnol. 26:49-52.

Davey M.G., Hedrick H.L., Bouchard S., Adzick N.S., Flake A.W. \& Doolin E.J. 2003. Computer-Assisted Stereology: point fraction of lung parenchyma and alveolar surface density in fetal and newborn sheep. Scanning 25:37-44.

DiDio L.J.A. \& Wakefield W. 1972. Origin, classification, nomenclature and incidence of the atrial arteries in normal human hearts, with special reference to their clinical importance. Acta Cardiol. 27(5):565-595.

DiDio L.J.A., Lopes A.C., Caetano A.C. \& Prates J.C. 1995. Variations 
of the origin of the artery of the sinoatrial node in normal human hearts. Surg. Radiol. Anat. 17(1):19-26.

Duerstock B.S., Bajaj C.L. \& Borgens R.B.A. 2003. A comparative study of the quantitative accuracy of tree-dimensional reconstructions of spinal cord from serial histological sections. J. Microscopy 210(2):138148.

Eliska O. \& Eliskova M. 1990. Arterial supply of the region of the coronary sinus in man. Cardiology 77(1):1-8.

Futami C., Tanuma K., Tanuma Y. \& Saito T. 2003. The arterial blood supply of the conducting system in normal human hearts. Surg. Radiol. Anat. 25(1):42-49.

Glomset D. \& Glomset A.T.A. 1940. A morphologic study of the cardiac conduction system in ungulates, dog and man. Part I. The sinoatrial node. Am. Heart J. 20:389-398.

Gundersen H.J. \& Jensen E.B. 1987. The efficiency of systematic sampling in stereology and its prediction. J. Microscopy 147:219-223.

Gundersen H.J., Jensen E.B., Kieu K. \& Nielsen J. 1999. The efficiency of systematic sampling in stereology-reconsidered. J. Microscopy 193:199-211.

Halpern M.H. 1955. The sino-atrial node of the rat heart. Anat. Rec. 123:425-436.

James T.N. 1961. Anatomy of the human sinus node. Anat. Rec. 141(1):109-135.

James T.N. 1977. The sinus node. Am. J. Cardiol. 40(6):965-986.

Jatene M.B., Hervoso C.M., Terra R.M., Guimarães M.H., Monteiro R., Jatene F.B. \& Jatene A.D. 1999. Importância da anatomia da circulação coronária atrial na operação de Cox para controle da fibrilação atrial. Revta. Bras. Cir. Cardiovasc. 14(1):32-38.

Jensen E.B.V. 1998. Local Stereology. $1^{\text {a }}$ ed. World Scientific, London. 247p.

Kawashima T. \& Sasaki H. 2003. The morphological significance of the human sinuatrial nodal branch (artery). Heart Vessels 18(4):213-219.

Kennel A.J. \& Titus J.L. 1972. The vasculature of the human sinus node. Mayo Clin. Proc. 47:556-561.

Kroustrup J.P. \& Gundersen H.J. 2001. Estimating the number of complex particles using the conneulor principle. J. Microscopy 203:314-320.

Krupa U. 1993. The sinuatrial nodal artery in the human heart. Folia Morphol. (Warsz) 52(1):29-37.

Krupa U. 1995. Vascularization of atria of the human heart in relation to the type of coronary ramification. Folia Morphol. (Warsz) 54(2):111122.

Kubinova L., Janacek J., Ribarc S., Cebasek V. \& Erzen I. 2001. Threedimensional study of the capillary supply of skeletal muscle fibres using confocal microscopy. J. Muscle Res. Cell Motility 22:217-227.

Kyriakidis M.K., Kourouklis C.B., Papaioannou J.T., Christakos S.G., Spanos G.P. \& Avgoustakis D.G. 1983. Sinus node coronary arteries studied with angiography. Am. J. Cardiol. 51(5):749-750.

Lopes A.C., Di Dio L.J.A. \& Buffolo E. 1998. Anatomical and clinical aspects of the blood supply of the sinoatrial node. Revta Assoc. Med. Bras. 44(1):47-49.

Mandarim-de-Lacerda C.A. 1990. Anatomia do Coração: clínica e cirurgia. Revinter, Rio de Janeiro. 133p.

Mandarim-de-Lacerda C.A. 2003. Stereological tools in biomedical research. Anais Acad. Bras. Ciênc. 75(4):469-486.

Mandarim-de-Lacerda C.A., Hidden G. \& Hureau J. 1985. Morfologia do nó sino-artial e de sua vascularização no coração de natimorto de termo. Arq. Bras. Cardiol. 44(2):107-109.

Mandarim-de-Lacerda C.A. \& Hureau U.J. 1986. Arterial vascularization of the sino-atrial and átrio-ventricular nodes and frequency of coronary artery branches in Papio erxleben, 1777. Folia Primatol (Basel) 46(2):65-69.
Mandarim-de-Lacerda C.A. \& Pereira L.M. 2001. Renal cortical remodelling by no-synthesis blockers in rats is prevented by angiotensin-converting enzyme inhibitor and calcium channel blocker. J. Cell. Molecul. Med. 5:276-283.

Mayhew T.M. 1992. A review of recent advances in stereology for quantifying neural structure. J. Neurocytol. 21:313-328.

Melo S.R. 2000. Estudo estereológico, histológico e ultra-estrutural do nó sinoatrial em ratos adultos e velhos. Tese de Doutorado, Instituto de Ciências Biomédicas, Universidade de São Paulo, São Paulo. 64p.

Melo S.R., Mandarim-de-Lacerda C.A. \& Sousa R.R. 2002. Características Ultra-Estruturais do nó sinoatrial de rato Wistar. Acta Sci. 24(3):681-685.

Melo-da-Silva A. \& Mandarim-de-Lacerda C.A. 1994. Morfologia e reconstrução anatômica do nó e da artéria do nó sinoatrial no coração de Rattus norvegicus. Revta Bras. Ciênc. Morfol. 11:82-87.

Nerantzis C. \& Avgoustakis D. 1980. An S-shaped atrial artery supplying the sinus node area: an anatomical study. Chest. 78(2):274-278.

Ovèina F. 2002. Vascularization of the sinoatrial segment in the heart conduction system in bovine and canine hearts. Med. Arh. 56(3):123125.

Ovèina F. \& Æemerliæ D. 1997. Clinical importance of intramural blood vessels in the sino-atrial segment of the conducting system of the heart. Surg. Radiol. Anat. 19(6):359-363.

Pakkenberg B. \& Gundersen H.J. 1988. Total number of neurons and glial cells in human brain nuclei estimated by disector and fractionator. J. Microscopy 150:1-20.

Pakkenberg B. \& Gundersen H.J. 1989. New Stereology method for obtaining unbiased and efficient estimates of total nerve cell number in human brain areas. Acta Pathol. Microbiol. Immunol. Scand. 97:677681.

Pina J.A.E., Pereira A.T. \& Ferreira A. 1975. Dos S. travaux originaux original works: vascularisation arterielle du nœud sino-auriculaire du cœur chez le chien [Arterial vascularization of the sino-auricular node of the heart in dogs]. Acta Cardiol. 30(2):67-77.

Randi R.E. 1988. Contribuição ao estudo da irrigação do nó sinoatrial em cães da raça Boxer. Tese de Doutorado, Faculdade de Medicina Veterinária e Zootecnia, Universidade de São Paulo, São Paulo. 40p.

Severino R.S. 1986. Contribuição ao estudo da vascularização arterial do nós sinoatrial em bovinos da raça Indubrasil. Dissertação de Mestrado, Faculdade de Medicina Veterinária e Zootecnia, Universidade de São Paulo, São Paulo. 66p.

Severino R.S., Carneiro e Silva F.O., Drummond S.S., Bombonato P.P. \& Alves F.P. 2000. Irrigação do nó sinoatrial em suínos da raça Piètrain. Braz. J. Vet. Res. Anim. Sci. 37(4):13-16.

Sow M.L., Ndoye J.M. \& Lo E.A. 1996. The artery of the sinuatrial node: anatomic considerations based on 45 injection-dissections of the heart. Surg. Radiol. Anat. 18(2):103-109.

Sterio D.C. 1984. The Unbiased estimation of number and sizes of arbitrary particles using dissector. J. Microscopy 134:127-136.

Truex R.C., Smythe M.Q. \& Taylor M.J. 1967. Reconstruction of the human sinoatrial node. Anat. Rec. 159(4):371-378.

Vidotti A.P. 2006. Vascularização arterial da região do nó sinoatrial em corações humanos normais. Tese de Doutorado, Faculdade de Medicina Veterinária e Zootecnia, Universidade de São Paulo, São Paulo. 97p.

Weibel E.R. 1979. Measuring through the microscope: development and evolution of stereological methods. J. Microscopy 155(3):393-403.

Weaver M.E., Pantely G.A., Bristow J.D. \& Ladley H.D. 1986. A quantitative study of the anatomy and distribution of coronary arteries in swine in comparison with other animals and man. Cardiovasc. Res. 20(12):907-917.

Wulfsohn D., Gundersen H.J., Jensen E.B. \& Nyengaard J.R. 2004. Volume estimation from projections. J. Microscopy 215:111-120. 